\title{
riccafd
}

Revista Iberoamericana de Ciencias de la Actividad Física y el Deporte

\section{EXPLORACIÓN BIOMECÁNICA DE LA CARRERA EN FUTBOLISTAS PROFESIONALES COLOMBIANOS: PROPUESTA INICIAL}

\section{BIOMECHANICAL EXPLORATION OF THE SPRINT OF SOCCER PLAYERS OF A COLOMBIAN PROFESSIONAL TEAM: AN INITIAL PROPOSAL}

Echavarria-Calderon, $\mathrm{M}^{1 \mathrm{ABC}}$; Galvis-Rincon, JC ${ }^{2 A C D}$

${ }^{1}$ Echavarria-Calderon, M. Fundación Universitaria de Ciencias de la Salud, Colombia mechavarria@fucsalud.edu.co

${ }^{2}$ Galvis-Rincon, JC. Fundación Universitaria de Ciencias de la Salud, Colombia jcgalvisr@gmail.com

\section{Responsabilidades}

ADiseño de la investigación

${ }^{B}$ Recolector de datos

CRedactor del trabajo

DIdea original y coordinador de toda la investigación

Financiación: Fundación Universitaria de Ciencias de la Salud y el Laboratorio de Análisis de Movimiento Movylab en la Ciudad de Bogotá, Colombia.

Agradecimientos: División de Investigaciones de la Fundación Universitaria de Ciencias de la Salud en Bogotá-Colombia. Club Deportivo Patriotas F.C de Boyacá- Colombia

Recibido el 30 de mayo de 2019

Aceptado el 29 de octubre de 2019

Correspondencia: Marcela Echavarría Calderón. mechavarria@fucsalud.edu.co

DOI: http://dx.doi.org/10.24310/riccafd.2020.v9i1.8301

\section{RESUMEN}

Introducción: El fútbol es el deporte en equipo más popular alrededor del mundo, se estima que alrededor del $4 \%$ de la población mundial lo practica (1), su actividad involucra correr, patear, saltar, parar y realizar cambios de dirección en altas y medias velocidades. La biomecánica de la carrera es importante ya que permite mejorar la técnica, e impactar en la prevención de lesiones.

Objetivo: determinar las características biomecánicas de la carrera de los jugadores de un equipo de fútbol profesional. 
Métodos: estudio observacional descriptivo tipo serie de casos de la carrera en 12 jugadores de fútbol profesional. Se incluyeron jugadores de 18 a 35 años pertenecientes al equipo sin lesiones de rodilla, cadera o tobillo en las últimas 4 semanas.

Resultados: La mediana de la velocidad fue $7.44 \mathrm{~m} / \mathrm{s}$ [RIQ: $6,93-7,75 \mathrm{~m} / \mathrm{s}$ ] siendo mayor en la pierna derecha $7.49 \mathrm{~m} / \mathrm{s}$ [RIQ 6,88 - 7,74 m/s] que en la izquierda. La mediana de la longitud de zancada, fue 3,21 m (RIQ 3,05-3,64 m) con predominancia en la pierna derecha $3.20 \mathrm{~m}$ [RIQ 3,11-3,74 m]. Se evidenció que el jugador de la posición volante fue quien mayor velocidad presentó, mientras que el volante derecho fue quien presentó la menor velocidad del grupo evaluado.

Conclusión: El estudio biomecánico de los futbolistas profesionales permite una caracterización del gesto deportivo de la carrera, permitiendo una visión diferente a las actualmente utilizadas tanto para la mejora en el rendimiento y estrategias de entrenamiento, como en la posible intervención en la prevención de lesiones.

Palabras clave: fútbol, fenómenos biomecánicos, carrera, medicina del deporte, análisis de sistemas.

\section{ABSTRACT}

Introduction: Football Soccer is the most popular team sport around the world, it is estimated that around $4 \%$ of the world's population practices it (1), this activity involves running, kicking, jumping, stopping and making changes of direction in high speed or half speed. The biomechanics of the sprint is important because it allows improving the technique and impact on the prevention of injuries.

Objective: To determine the biomechanical characteristics of the sprint of professional soccer team players.

Methods: descriptive observational study of a series cases of the sprint in 12 professional soccer players. We included players from 18 to 35 years old belonging to a professional team without knee, hip or ankle injuries in the last 4 weeks.

Results: The median speed was $7.44 \mathrm{~m} / \mathrm{s}$ [RIQ: $6.93-7.75 \mathrm{~m} / \mathrm{s}$ ] being higher in the right leg $7.49 \mathrm{~m} / \mathrm{s}$ [RIQ $6.88-7.74 \mathrm{~m} / \mathrm{s}$ ] than on the left. The median stride length was $3.21 \mathrm{~m}$ (RIQ 3.05-3.64 $\mathrm{m}$ ) with predominance in the right leg $3.20 \mathrm{~m}$ [RIQ 3.11-3.74 m]. It was evidenced that the winger player was the one who presented the highest speed, while the middle forward was the one who presented the lowest speed of the group evaluated.

Conclusion: The biomechanical study of professional soccer players allows a characterization of the sports gesture of the sprint, allowing a different vision to those currently used both for the improvement in performance and training strategies, as well as in the possible intervention in the prevention of injuries. 
key words: soccer, biomechanical phenomena, running, systems analysis, sports medicine

\section{INTRODUCCIÓN}

El fútbol es el deporte en equipo más popular alrededor del mundo, con 265 millones de jugadores entre hombres y mujeres, $4 \%$ de la población mundial juegan fútbol (1). Su actividad involucra correr, patear, saltar, parar y realizar cambios de dirección en altas y medias velocidades, lo que puede conducir a un número considerable de lesiones (2), lo que hace importante conocer el patrón biomecánico de cada uno de ellos.

Por ser uno de los deportes más dinámicos, complejos y demandantes es caracterizado por un número significativo de duelos entre los oponentes que requiere una excelente preparación: motriz, táctica, técnica, y mental (3). La preparación de un jugador ésta frecuentemente enfocada en el mejoramiento de la técnica o habilidades tácticas a expensas de desarrollar talento motriz (3).

Como muchos otros deportes en equipo, el fútbol involucra también un número variado de posiciones en el campo de juego con diferentes requerimientos físicos (3). Cada equipo se conforma de 11 jugadores, de los cuales 1 será el portero o arquero encargado de cubrir la portería y evitar el gol y los demás estarán repartidos entre atacantes y defensores según la disposición táctica del cuerpo técnico (4).

Actualmente hay varios estudios que han analizado la composición y destrezas de los jugadores durante los partidos y entrenamientos, encontrando que en promedio, durante una temporada los jugadores realizan una caminata de $620 \mathrm{~km}$ con un promedio por partido de $3 \mathrm{~km}$ y entrenamiento de $2 \mathrm{~km}$, (5). Así mismo, en cuanto al trote, el promedio durante la temporada fue de $1180 \mathrm{~km}$ que corresponde a $5 \mathrm{~km}$ por partido y $4 \mathrm{~km}$ por sesión de entrenamiento (5). Respecto al Sprint o carrera, el promedio fue de $0,7 \mathrm{~km}$ durante un partido y 1,5 $\mathrm{km}$ durante un entrenamiento para un promedio general de $372 \mathrm{~km}$ (5). El promedio de carrera con balón está estimado en $100 \mathrm{~km}$ siendo $0.2 \mathrm{~km}$ durante un partido y 0,4 km durante el entrenamiento (5), lo que sugiere que la carrera es un factor importante de exposición que puede llevar a lesiones tanto de índole traumático como por uso excesivo.

\section{Biomecánica de la Carrera}

La carrera hacia adelante se compone de un patrón específico que se podría dividir en 4 fases: arranque (desde el punto cero hasta que da el primer paso), aceleración (desde el primer paso hasta que alcanza la velocidad máxima), máxima velocidad (desde que alcanza el máximo desplazamiento en el tiempo hasta que inicia el frenado) y desaceleración (desde que inicia el descenso de la velocidad hasta que se detiene) (6). 
Cada una de estas fases, tiene componentes para su desarrollo. La zancada es aquella comprendida entre los apoyos de ambas piernas, es decir, la distancia entre 2 fases sucesivas acabadas por la misma pierna, y para su desarrollo se puede definir 3 fases: impulsión (fase inicial, en donde el centro de masa se localiza hacia anterior, es en la que se hace el empuje), sustentación (fase de transición donde el centro de masa es vertical), amortiguación (el centro de masa está hacia posterior, es la fase en la que se realiza el frenado) (7).

Por su parte, el paso se define como la distancia entre 2 puntos sucesivos de contacto de pies opuestos, podría considerarse entonces, que el paso se compone de las mismas fases de la zancada pero vistos desde una misma pierna (unilateral).

Entender la biomecánica de la carrera es importante ya que permite mejorar la técnica, e impactar en la prevención de lesiones, debido a que la carrera es una habilidad motriz básica, es decir, una actividad común que se desarrolla en la infancia la cual está fundamentada en aprendizajes motrices durante el desarrollo neurológico, es por esto que el presente proyecto busca determinar cuáles son las características biomecánicas de la carrera de los jugadores de un equipo de fútbol profesional colombiano de la primera división (Patriotas F.C).

\section{MATERIAL Y METODOS}

\section{Diseño de la Investigación}

Se realizó un estudio observacional descriptivo tipo serie de casos, en dónde se determinaron variables biomecánicas relacionadas con la carrera en 12 jugadores del equipo de fútbol profesional de Patriotas F.C.

\section{Tipo y estrategia de Muestreo}

Los jugadores fueron seleccionados por el cuerpo técnico para asistir a la valoración, quienes se presentaron por sus propios medios en el laboratorio y accedieron de manera voluntaria a la evaluación como parte de la pretemporada 2019-1. Al ser un estudio descriptivo no se requirió un tamaño de muestra.

\section{Criterios de Selección}

Se incluyeron jugadores entre 18 años y 35 años, de sexo masculino, pertenecientes un equipo de fútbol profesional colombiano que se encuentren haciendo parte del equipo de la primera división (Patriotas F.C). Se excluyeron aquellos jugadores con antecedente de incapacidad por lesión de cadera, rodilla y/o tobillo en las últimas cuatro semanas y disminución de arcos de movimiento articular de cadera, rodilla y/o tobillo. 


\section{Análisis de Movimiento}

Los datos fueron recolectados en el laboratorio de análisis de movimiento (MovyLab) del Hospital Universitario Infantil de San José (HUISJ) en Bogotá, Colombia, a partir del sistema de ocho cámaras VICON Vantage ${ }^{\text {, el cual, es }}$ un modelo que calcula la cinemática de la articulación y la cinética de las posiciones, como también las medidas antropométricas de los marcadores específicos en el individuo (8). Previo al inicio de la recolección, se hizo una evaluación de los jugadores por el médico especialista (deportólogo) para determinar la aptitud teniendo en cuenta los criterios de inclusión y exclusión con registro de historia clínica y aquellos que cumplen con dicho requisito se les explicó el estudio, sus riesgos y sus beneficios, se resolvieron dudas y se les entregó el consentimiento informado para su lectura, aprobación y firma.

Los datos de biomecánica fueron tomados a través del examen computarizado del sistema del laboratorio MovyLab, siguiendo estándares para el posicionamiento de los marcadores y la toma de datos. Cada jugador realizó 4 intentos de carrera a máxima velocidad en un espacio de evaluación de 15 metros en donde se describirán la distancia recorrida por unidad de tiempo (velocidad de carrera), el número de pasos por unidad de tiempo (cadencia), la distancia entre 2 puntos sucesivos de contacto de pies opuestos (longitud de paso), la diferencia entre 2 fases sucesivas acabadas por la misma pierna (longitud de zancada), la duración de las 2 fases sucesivas acabadas por la misma pierna (tiempo de zancada), y el tiempo entre 2 puntos sucesivos de contacto de pies opuestos (tiempo de paso). Para el análisis de los datos se tomó el mejor intento basado en la velocidad.

\section{Plan de análisis}

Para llevar a cabo los objetivos de caracterización las variables cualitativas se analizarán a partir de frecuencias y porcentajes. Para las variables cuantitativas inicialmente se comprobó si se la distribución era normal por medio de la prueba de Shapiro Wilk, si se distribuían normal se representaron las variables con medias y desviaciones estándar con sus respectivas gráficas de histogramas. Por otra parte, si no fue normal se utilizó medianas y rangos intercuartiles representándolo gráficamente con diagramas de cajas y alambres.

\section{Consideraciones Éticas}

La aplicación del examen biomecánico y los datos recolectados durante la investigación se manejaron con total confidencialidad según las normas de ética médica en Colombia, bajo la ley 23 de 1981 y siguiendo la resolución número 8430 de 1993 del ministerio de salud de Colombia. Se consideró una investigación de bajo riesgo. El protocolo fue aprobado por el comité de investigaciones de la Fundación Universitaria de Ciencias de la Salud y contó con la aprobación del comité de ética del Hospital Universitario Infantil de San José en la ciudad de Bogotá. 


\section{RESULTADOS}

En el análisis biomecánico de los 12 jugadores, se encontró que la mediana de la velocidad fue de $7.44 \mathrm{~m} / \mathrm{s}$ [RIQ: $6,93-7,75 \mathrm{~m} / \mathrm{s}$ ] siendo mayor en la pierna derecha que en la izquierda $7.49 \mathrm{~m} / \mathrm{s}$ [RIQ $6,88-7,74 \mathrm{~m} / \mathrm{s}$ ] y $7.25 \mathrm{~m} / \mathrm{s}$ [RIQ 6,97-7,75 $\mathrm{m} / \mathrm{s}$ ] respectivamente (Figura 1). Al evaluar la longitud de zancada, se encontró que la mediana fue de 3,21 m (RIQ 3,05-3,64 m) con predominancia en la pierna izquierda 3,28 m [RI 2,97-3,59 m] respecto a la derecha $3.20 \mathrm{~m}$ [RIQ 3,11-3,74 m]. La cadencia mediana general fue de 264 pasos/min (RIQ: 249-284pasos/min) con un tiempo mediano de paso de 0.22 segundos (RIQ: $0.21-0,24$ segundos) y una mediana de tiempo de zancada de 0.46 segundos (RIQ: 0,42-0,48 segundos). La Figura 1 muestra los parámetros evaluados en la pierna derecha e izquierda de los 12 jugadores del estudio biomecánico de la carrera.

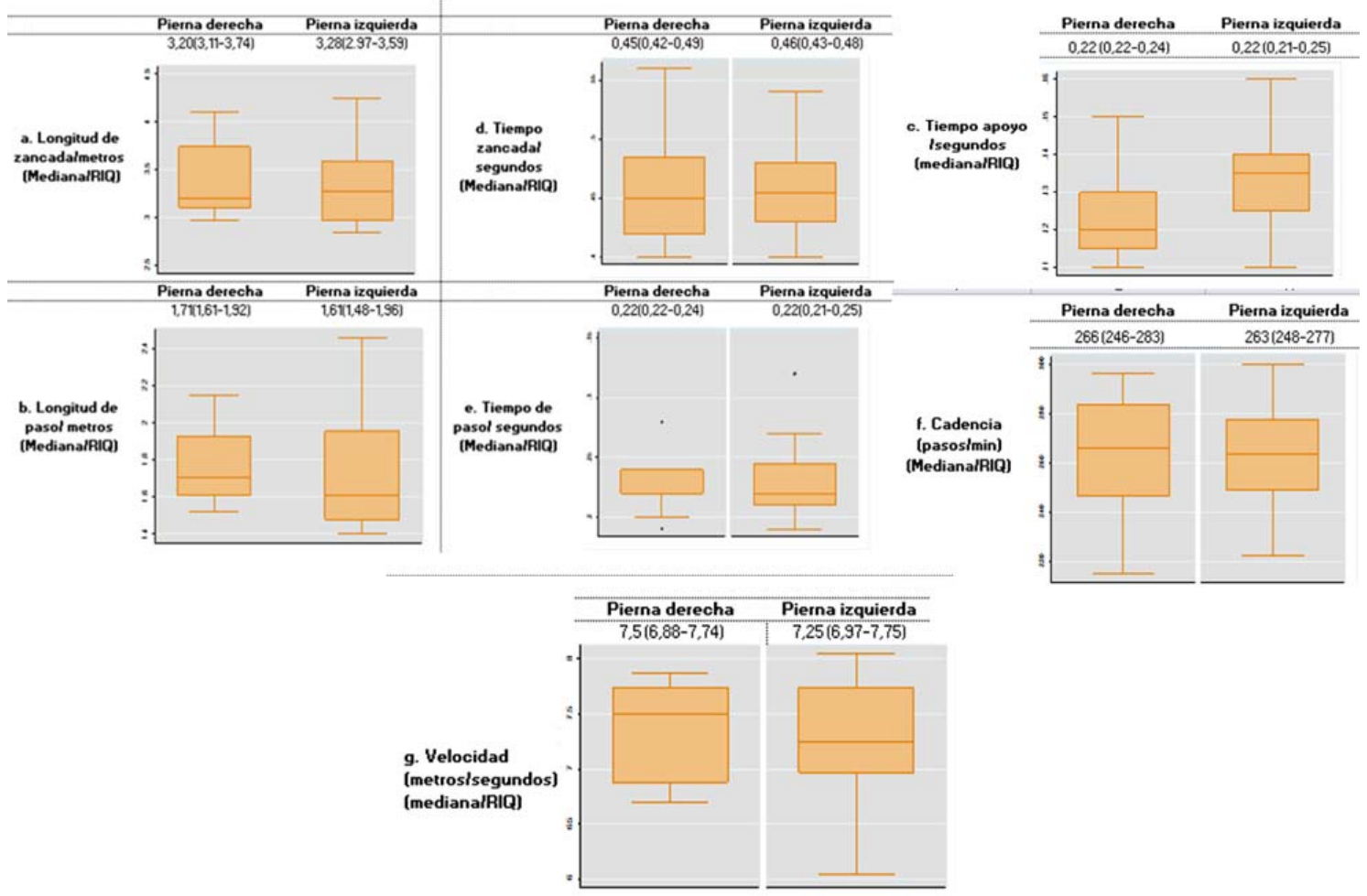

Figura 1. Evaluación General de la Carrera.

Evaluación de mediana de a. Longitud de zancada, b. longitud de paso, c. tiempo de apoyo, d. tiempo de zancada, e. tiempo de paso, f. cadencia y g. velocidad para la pierna derecha, izquierda RIQ: rango intercuartílico. M: metros. S: Segundos. M/S: metros/segundos. Pasos/min: Pasos/minutos.

En cuanto a la velocidad según la posición en el campo de cada uno de los jugadores evaluados, se encontró que el jugador en posición volante (quien es un jugador que se ubica por estrategia táctica en el medio campo y se desplaza tanto por derecha como por izquierda) fue quien mayor velocidad presentó, mientras que el volante derecho (el jugador que se mueve en el medio campo pero hacía el lado derecho) fue quien presentó la menor velocidad. 
Al analizar la relación de la longitud de la zancada con la velocidad en cada pierna, teniendo en cuenta la posición en el campo de cada uno de los 12 jugadores, se observó como a medida que la longitud de la zancada aumenta, la velocidad es mayor. Igualmente, la gráfica de la pierna derecha tiene una tendencia a una pendiente mayor respecto a la pierna izquierda, y que el jugador con menor rendimiento fue el mismo tanto para la pierna derecha como izquierda (volante derecho). (Figura 2.)

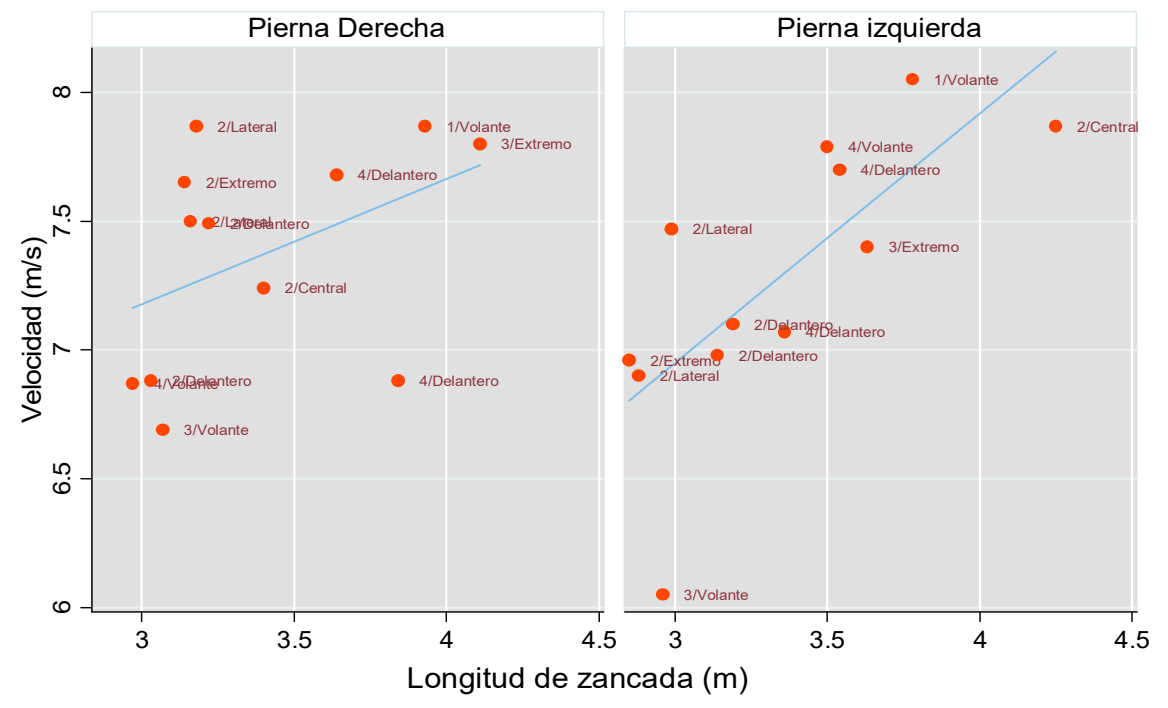

Figura 2. Velocidad en relación con la Longitud de Zancada.

La longitud de paso tiene un comportamiento similar a la longitud de zancada, mostrando una gráfica de regresión lineal con pendiente menor en la pierna derecha al comparar con la pierna izquierda y de igual forma, el jugador con menor rendimiento en cuanto a longitud de paso versus velocidad es el volante (volante derecho) (Figura 3).

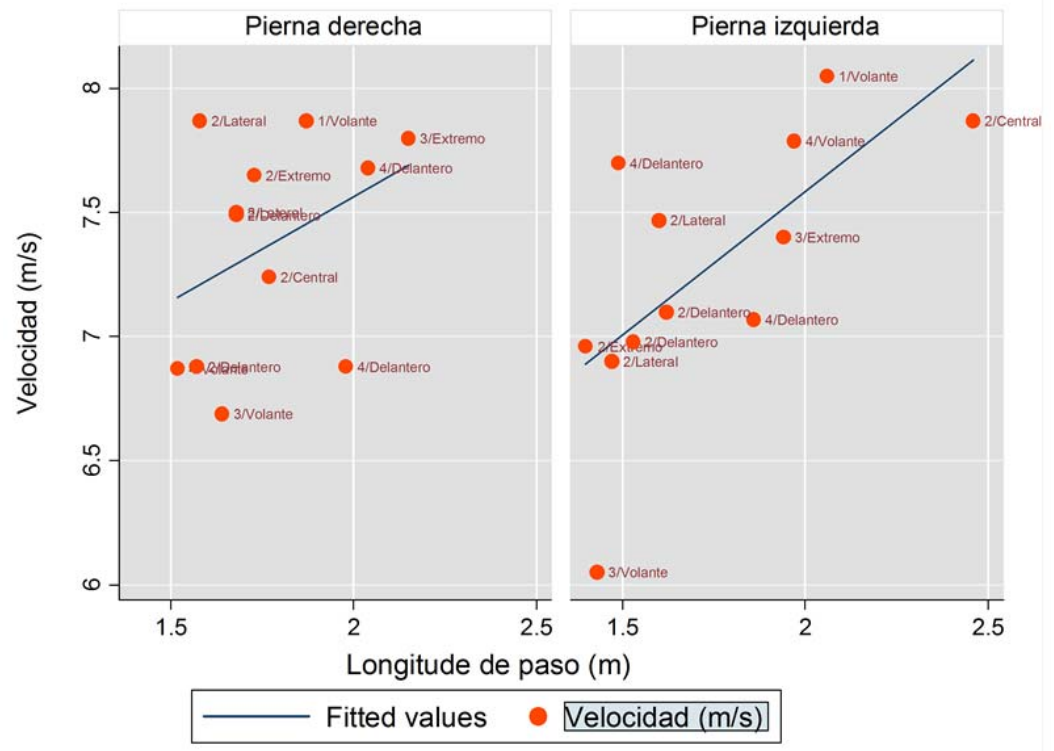

Figura 3. Velocidad en relación con la Longitud de Paso. 


\section{DISCUSIÓN}

El fútbol es uno de los deportes con mayor impacto en nuestro país y en el mundo debido a la gran acogida tanto profesional como aficionado, con un sinnúmero de seguidores (1); es por esto que se vuelve de vital importancia conocer los principales gestos que componen el deporte, entre ellos la carrera, con el fin de establecer pautas para mejorar el rendimiento y disminuir la prevalencia de lesiones. En otras palabras, conocer los gestos propios del deporte y sus condiciones biomecánicas, permite hacer prevención primaria, rehabilitación y retorno al juego.

El análisis biomecánico se encarga del estudio del movimiento corporal y de las fuerzas que lo producen como la inercia, gravedad y contracción muscular que se ejecutan a partir de las articulaciones (9) y comprende la mecánica de los seres vivos con el entendimiento del conocimiento de las fuerzas que producen el movimiento, así como el soporte anatómico, iniciación neuronal, control integrado, percepción y su diseño central (10). Todo lo anterior es posible analizarlo en un gesto determinado como el resultado de las integraciones de los diferentes sistemas, en el caso del artículo actual, la carrera.

Los resultados concuerdan con datos descritos en atletas, por ejemplo, a mayor velocidad de carrera, en la zancada, la fase de vuelo va a ser más larga y la fase de apoyo más corta, logrando una amplitud de zancada mayor, en otras palabras, a mayor velocidad mayor longitud de zancada, como lo describe Mackala (11), nuestros deportistas alcanzaron una mayor velocidad cuando realizaban una longitud de zancada mayor.

Al evaluar los datos de la zancada respecto a la velocidad, el presente estudio encontró que el deportista con menor rendimiento era el mismo tanto para la pierna derecha como la izquierda y que coincide con el desenlace final de velocidad, siendo el menos veloz, Esto podría ser explicado por la longitud de las extremidades, sin embargo dicha variable no fue tenida en cuenta para el análisis de los datos, de igual forma, es posible explicar este comportamiento por fallas en la técnica de carrera que obligarían a hacer intervenciones para mejorar el rendimiento deportivo y disminuir el riesgo de lesión (Figura 2).

Respecto a la longitud de paso, al ser un componente de la zancada, tiene un comportamiento similar cuando se relaciona con la velocidad, lo que permite entender que a medida que el paso es más largo, se favorece la velocidad y por tanto la amplitud de la zancada. La figura 2 y 3 muestra una regresión lineal del análisis de la zancada y el paso, con comportamiento diferente, que puede ser explicado por factores externos como la fuerza muscular o el movimiento angular de las articulaciones, los cuales no fueron evaluados en el presente estudio.

Si se combinan todos los factores mencionados (longitud de paso, longitud de zancada, velocidad) y se relacionan con el tiempo de apoyo de cada una de las piernas durante la carrera, es posible pensar que un menor tiempo de apoyo conlleva a un mayor rendimiento en la carrera. Como se menciona en la litera- 
tura, la fase de apoyo tiene como objetivo impulsar $(6,7)$, logrando una mejoría en la velocidad, lo que lleva a concluir que si el tiempo de fricción es menor pero efectivo, mejorará el rendimiento de la carrera.

Ahora bien, hablando de la cinemática de la carrera, se encuentran estudios evaluando diferencias biomecánicas entre la extremidad dominante y la no dominante y sus implicaciones en el rendimiento (12). El actual estudio muestra que la extremidad dominante coincide con la mejor velocidad sólo en 4 de los deportistas evaluados. Por su parte, la longitud de la zancada es mayor en aquella pierna que es más veloz en 9 de los 12 futbolistas analizados, lo que sugiere que el lado izquierdo y el lado derecho se comportan de manera diferente, sin relación con la dominancia. La Figura 2 y 3 muestra el comportamiento de la velocidad en relación con la longitud de zancada y longitud de paso para cada una de las extremidades; observamos que existe una diferencia en el comportamiento entre la velocidad y por lo tanto la longitud entre lo realizado por el lado derecho y el izquierdo; esto sugiere que se podría considerar un modelo de entrenamiento dirigido a la ambidiestralidad, teniendo en cuenta que la carrera es una habilidad motriz básica y podría ser entrenada desde fases tempranas del desarrollo neuromotriz o en entrenamientos específicos para deportistas con el fin de mejorar el rendimiento en el campo.

Dentro de las limitaciones del estudio, se encuentra el bajo número de la muestra que podría implicar un sesgo de medición, así mismo sólo se tuvo en cuenta el gesto de la carrera, y fue evaluada en un espacio controlado y monitorizado y no en el entorno normal de práctica deportiva por lo que los investigadores recomiendan, realizar nuevos estudios donde se evalúe un mayor número de jugadores así como la medición de otros gestos deportivos y tener en cuenta las condiciones del campo e indumentaria deportiva que más se asemeje a la práctica general principalmente para los programas de prevención de lesiones.

En conclusión, El presente estudio describe el gesto de la carrera realizado en un entorno controlado con base en un estudio computarizado biomecánico en laboratorio a futbolistas profesionales y con diferente posición en el campo con el fin de establecer el punto de partida para el conocimiento del comportamiento biomecánico en una población específica. Es un preámbulo para el estudio biomecánico de los futbolistas profesionales, permitiendo una caracterización del gesto deportivo de la carrera en estos deportistas, permitiendo una visión diferente a las actualmente utilizadas tanto para la mejora en el rendimiento y estrategias de entrenamiento, como por ejemplo el entrenamiento de la ambidiestralidad desde fases tempranas del desarrollo motriz como en programas específicos de alto rendimiento para mejorar el rendimiento en el campo, así como en la posible intervención en la prevención de lesiones.

\section{LIMITACIONES Y CAMINOS FUTUROS}

Dentro de las limitaciones del estudio, se encuentra el bajo número de la muestra que podría implicar un sesgo de medición, así mismo sólo se tuvo en 
cuenta el gesto de la carrera, y fue evaluada en un espacio controlado y monitorizado y no en el entorno normal de práctica deportiva por lo que los investigadores recomiendan, realizar nuevos estudios donde se evalúe un mayor número de jugadores así como la medición de otros gestos deportivos y tener en cuenta las condiciones del campo e indumentaria deportiva que más se asemeje a la práctica general principalmente para los programas de prevención de lesiones.

\section{AGRADECIMIENTOS Y FINANCIACIÓN}

Agradecemos a la División de Investigaciones de la Fundación Universitaria de Ciencias de la Salud por su asesoría en la creación y desarrollo del proyecto. De igual forma, agradecemos al Laboratorio de Análisis de Movimiento Movylab por su apoyo en la toma e interpretación de datos, así como financiación como contrapartida del proyecto. Agradecemos al Club Deportivo Patriotas F.C, a sus directivos, al cuerpo técnico y a los jugadores por el apoyo brindado con el transporte y disponibilidad de tiempo de los jugadores para la obtención de los datos.

Ninguno de los investigadores declara conflictos de interés.

El presente proyecto recibió financiación de la Fundación Universitaria de Ciencias de la Salud y del laboratorio de Análisis de Movimiento Movylab.

\section{REFERENCIAS BIBLIOGRÁFICAS}

1. Daneshjoo A MA, Rahnama N, Yusof A. The effects of injury prevention warm-up programmes on knee strngth in male soccer players. Biology of Sport. 2013;30(4):281-8. eng.

2. Ibrahim A, Murrell GA, Knapman P. Adductor strain and hip range of movement in male professional soccer players. J Orthop Surg (Hong Kong). 2007 Apr;15(1):46-9. PubMed PMID: 17429117. eng.

3. Andrzejewski M, Chmura J, Pluta B, Kasprzak A. Analysis of motor activities of professional soccer players. J Strength Cond Res. 2012 Jun;26(6):1481-8. PubMed PMID: 22614138. eng.

4. (IFAB) TiFAB, FIFA. Reglas de Juego 2016/17. 2016.

5. Luhtanen P. Aspectos Biomecánicos del Rendimiento en el Fútbol PubliCE Standard 2004.

6. Mero A. Biomechanics of Sprint Running. In: P.V. Komi, Gregor. R, editors.: Journal of Sports Medicine 1992. p. 376-92.

7. Álvarez-Romero D. BIOMECHANICAL ASSESMENT OF SPRINT RUNNINIG. TRABAJO DE FIN DE GRADO EN CIENCIAS DE LA ACTIVIDAD FÍSICA Y DEL DEPORTE ed: Universidad de León; 2014.

8. VICON®. Plug-in-Gait modelling instructions. Vicon ${ }^{\circledR}$ Manual, Vicon $₫ 612$ Motion Systems Oxford, UK,. 2010.

9. Rueda-Peláez L. Principios de Biomecánica. Apunts Medicina de L’Esport. 2006;148:39-43. 
10. Izquierdo M. Biomecánica y bases neuromusculares de la Actividad Física y el Deporte. Buenos Aires2008. 761 p.

11. Mackala K. Optimisation of performance through kinematic analysis of the different phases of the 100 metres. New Studies in Athletics; 2007. p. 7-16.

12. H.C. Dörge, T. Bull Andersen, H. SØrense, Simonsen EB. Biomechanical differences in soccer kicking with the preferred and the non-preferred leg Journal of Sports Sciences; 2011. p. 293-9. 